\title{
IDENTIFICATION, CHARACTERIZATION AND QUANTIFICATION OF CHEMICAL COMPOUNDS IN SELECTED EDIBLE WILD LEAFY VEGETABLES
}

\author{
* Salisu, T. F. ${ }^{1}$, Okpuzor, J. E. ${ }^{1}$ and Jaja, S. I. ${ }^{2}$ \\ ${ }^{1}$ Department of Cell Biology and Genetics, University of Lagos, Akoka, Yaba, Lagos, Nigeria \\ ${ }^{2}$ Department of Physiology, College of Medicine, University of Lagos, Idi-Araba, Lagos, Nigeria \\ *Corresponding author's email: basstiscoa@gmail.com;Tel: +2348033732248
}

(Received: $7^{\text {th }}$ October, 2017; Accepted: $6^{\text {th }}$ February, 2019)

\section{ABSTRACT}

This study identified, characterized and quantified the constituents of Basella alba Linn. var. alba, Crassocephalum crepidioides (Benth.) S. Moore, Launaea taraxacifolia Amin Ex. C. Jeffrey (Wild lettuce), Senecio biafrae Oliv. \& Hiern. and Solanum nigrum L. var. virginicum (Black nightshade) leaves. Pulverised dried leaves (10 g each) of the vegetables were separately Soxhlet-extracted with $60 \mathrm{ml}$ of $\mathrm{n}$-hexane $(99 \%)$. Aliquots $(2 \mu \mathrm{l})$ of each concentrated plant extract were analysed using gas chromatography - mass spectrometry (GC-MS) technique. Forty-four (44) volatile compounds were identified from the analyses. Some of them are known antioxidants that are beneficial to health. Linoleic and palmitic acids were identified in leaves of $B$. alba (6.85\% and 5.47\%), C. crepidioides (14.88\% and $27.22 \%)$ and L. taraxacifolia $(20.78 \%$ and $42.35 \%)$ respectively. However, linoleic acid was absent in S. nigrum but linolenic acid (47.47\%) was present as the major fatty acid. Oleic acid was identified respectively in leaves of B. alba (2.37\%), C. crepidioides (6.86\%) and $L$. taraxacifolia $(7.14 \%)$. The information obtained from the GC-MS analysis of these wild underutilized vegetables can be used to develop novel drugs or food supplements.

Keywords: Underutilized Vegetables, GC-MS Analysis, Antioxidants

\section{INTRODUCTION}

Vegetables are abundant in nature, possessing promising nutritive and therapeutic values that can nourish the ever-increasing human population (Janick, 2011; Amujoyegbe et al., 2015). Despite their abundance, nutritional roles in diet and possible significance in the modulation of certain diseases such as heart disease, wild leafy vegetables are usually under exploited for research and food (Amujoyegbe et al., 2015). There are several varieties of these vegetables in the rural areas ranging from the wild to under cultivated or underutilized. Migration to urban centres has a great impact on the choice of vegetables used as food because underutilized vegetables are not readily available in our urban markets (Odukoya et al., 2007). These vegetables are often considered underutilized in terms of the scanty research attention with limited scientific information on them, their underexploited economic potential and lack of technologies for improved farming practices (Osewa et al., 2013).

Underutilized indigenous vegetables are naturally growing wild plant species, rarely cultivated and gradually becoming endangered vegetables in the natural ecosystem (Stamp et al., 2012). These vegetables are usually collected from fallows, watercourses, field margins, disturbed fields, protected home gardens, refuse hills and generally in abandoned areas. Most of these wild indigenous vegetables are edible, underexploited and have served as basis of nutritional livelihood and medicinal plants in the rural areas for several years. These neglected native plant species are still harvested from wild, uncultivated and less included in scientific research (Adebooye and Ajayi, 2008). They remain less utilized resulting in low production, processing, distribution, marketing, consumption and research input which are critical for proper integration into WHO's global initiative for fruit and vegetable consumption promotion (Smith and Eyzaguirre, 2007).

Increase in human activities is equally disturbing the existence, stability and natural regeneration of these vegetables (Shabu and Uchi, 2013). Consequently, these result in gradual loss of genetic diversity of the vegetables (Nnamani et al., 2010). The importance of wild leafy vegetables in 
folklore medicine cannot be undervalued due to their cost effectiveness, easy digestibility and efficacy.

Basella alba, a succulent green vegetable, commonly known as Ceylon spinach is a cool season plant which belongs to the family Basellaceae. It is locally called "Amunututu" (Yoruba) in southwestern Nigeria. The leaves are used traditionally as anti-hypertensive (Olowokudejo et al., 2008), anti-inflammatory (Kumar et al., 2011), antimicrobial, antioxidant (Suguna et al., 2015), anti-anaemic and for hepatoprotection (Bamidele et al., 2010), indicating the importance in ethnomedicine. Crassocephalum crepidioides (Asteraceae) also called "Ẹ fọ́ Ebòlò," (Yoruba), Babohoh (Hausa), Notị-Ėnē (Antelope's Ear) (Igbo), Red flower rag leaf or Fireweed (English), has been in use among folks in treating acute hepatitis and fever (Tomoyuki et al., 2005). Its antioxidant and hepatoprotective activities have also been reported in literature (Tomoyuki et al., 2005; $\mathrm{Ng}$ et al., 2012). Antioxidant and anticancer properties of Solanum nigrum (Solanaceae) leaves have also been well documented (Aboul-Enein et al., 2014). The plant has been used extensively in traditional medicine to treat various ailments such as pain, inflammation and fever.

Senecio biafrae (Compositae) is known for its therapeutic virtues, notably in Nigeria where it is used in the treatment of diabetes mellitus (Adebayo, 2009). Senecio biafrae leaves, "Worowo" (Yoruba), are used by traditional practitioners to treat infertility in women (Lienou et al., 2010), heart problems (Ayodele, 2005), as well as serve as antihypertensive (Olowokudejo et al., 2008). Hypolipidaemic, hypoglycemic and antioxidants properties of Launaea taraxacifolia (Asteraceae) plant, Ẹ fọ́ yánrin (Yoruba), have also been reported (Dansi et al., 2008; Obi, 2011; Arawande et al., 2013).

However, standardization of the plant preparation, identification and characterization of the specific chemical components in the plant are required for optimum nutritional and therapeutic potentials. Furthermore, these wild leafy vegetables could promote socio-economic empowerment of communities where they are found and industrially exploited (Ogunwusi and Ibrahim, 2016). Considering this view, the present study was aimed to identify, characterize and quantify the chemical constituents of these wild selected leafy vegetables using gas chromatography mass spectrometry (GC-MS) technique.

\section{MATERIALS AND METHODS}

Plant Materials : The leaves' extracts of the following plants were studied

a. Basella alba Linn. var. alba

b. Crassocephalum crepidioides (Benth.) S. Moore

c. Launaea taraxacifolia. Amin Ex. C. Jeffrey

d. Senecio biafrae Oliv. \& Hiern.

e. Solanum nigrum L. var. virginicum

\section{Collection of Plant Materials}

Fresh tender leaves of B. alba (LUH5807A), C. crepidioides (LUH1229A), L. taraxacifolia (LUH5806A), S. biafrae (LUH1227A) and S. nigrum (LUH1228A) were collected from Titi's home garden, Ota, Ogun state (Latitude $6.7^{\circ} \mathrm{N}$ and longitude $3.2^{\circ} \mathrm{E}$ ), Ikoro market, Ikoro, Ekiti (Latitude $7.8^{\circ} \mathrm{N}$ and Longitude $5.0^{\circ} \mathrm{E}$ ), and Kajola, Ibadan, Oyo state (Latitude $7.5^{\circ} \mathrm{N}$ and Longitude $3.9^{\circ} \mathrm{E}$ ) south-western Nigeria, during the rainy season (June - August, 2014). The samples were authenticated by a taxonomist in the Botanical Herbarium, Dr. A. B. Kadiri of the Department of Botany, Faculty of Science, University of Lagos, Lagos, Nigeria. A voucher specimen for each plant was deposited in the Botanical Herbarium of the Department of Botany, Faculty of Science, University of Lagos, Nigeria where voucher numbers indicated in the brackets were obtained.

\section{Preparation of Plant Samples}

Fresh B. alba, C. crepidioides, L. taraxacifolia, S. biafrae and $S$. nigrum leaves were separately washed thoroughly with clean water and air-dried for a week under the shade at room temperature. Then the dried leaves of each of the samples were coarsely pulverized using electric blender and kept 
in air tight bottle before extraction for GC-MS analysis.

\section{Soxhlet Extraction of Vegetables for GC-MS Analysis}

The Soxhlet extraction process was carried out using $60 \mathrm{ml}$ of $\mathrm{n}$-hexane $(99 \%)$ as an extractant. Solvent was poured into the round bottomed extraction flask (capacity $100 \mathrm{ml}$ ), weighed and placed on the heating mantle. After this, the thimble containing the dried ground plant sample $(10 \mathrm{~g})$ was placed into the extraction chamber of the Soxhlet extractor. Lastly, the condenser was placed on top of the extractor and all the parts were fixed vertically. The extraction was carried out for three intervals of time, which are 3 hours, 6 hours and 9 hours (Ahmad et al., 2009). The extracts were concentrated to $\sim 2 \mathrm{ml}$ using nitrogen concentrator. Aliquot $(2 \mu \mathrm{l})$ of each $n-$ hexane-concentrated crude plant extract was injected into the split-less GC-MS system for analysis.

\section{Gas Chromatography-Mass Spectrometry (GC-MS) Analysis of Bioactive Components of Vegetables Extracts}

Gas Chromatography-Mass Spectrometry (GCMS) analyses were carried out using 7890A Gas chromatography system coupled to VL/MSD 5975C mass spectrometer (GC-MS Agilent Technologies, Santa Clara, USA) instrument employing the following conditions: Column HP5MS fused silica capillary column $[30 \mathrm{~m}$ (length) x $0.32 \mathrm{~mm}$ (diameter) x $0.25 \mu \mathrm{m}$ (film thickness)] composed of 100\% dimethyl polysiloxane. Helium gas $(99.99 \%)$ was used as the carrier gas at constant flow rate of $1 \mathrm{ml} / \mathrm{min}$ and an injection volume of $1 \mu \mathrm{l}$ was employed with injector temperature at $250{ }^{\circ} \mathrm{C}$ and pressure at $8.802 \mathrm{psi}$. The oven temperature was programmed initially from $80{ }^{\circ} \mathrm{C}$ (held for 2 minutes) with an increase of $5{ }^{\circ} \mathrm{C} / \mathrm{min}$. to $120{ }^{\circ} \mathrm{C} / \mathrm{min}$, then 10 ${ }^{\circ} \mathrm{C} / \mathrm{min}$ to $240{ }^{\circ} \mathrm{C} / \mathrm{min}$, to hold for $6 \mathrm{~min}$. The total GC running time for each sample was 30 min. The area under a peak accurately represents the quantity of the component present in each plant sample. Software adopted to handle mass spectra and chromatogram was a ChemStation. The interpretation of mass spectrum of GC-MS was done using the database of National Institute of Standard and Technology (NIST) version 2 year 2011 library. The mass spectrum of each of the unknown components was compared with the spectrum of the known components stored in the NIST library to ascertain the name, molecular weight and structure of the components of each of the vegetables' extracts.

\section{RESULTS}

\section{GC-MS Analysis of Components of Vegetables Extracts}

The compounds present in $\mathrm{n}$-hexane extracts of B. alba, C. crepidioides, L. taraxacifolia, S. biafrae and $S$. nigrum leaves were identified by GC-MS method. The chromatograms of vegetable extracts analysed are presented in figures 1 to 5 . Each peak represents a compound with different quantification and quality based on percentage by ChemStation's calibration mode (Software). Some peaks depict the same compound with different retention time and thus their area percentage compositions are additive. The GC-MS analysis of these vegetables extracts resulted in identification and quantification of 44 compounds. The identified compounds with their molecular formula, molecular weight (MW), retention time (RT), area percentage composition (quantity) and quality (matching factor) of each vegetable extract are presented in tables 1 to 5 respectively. 
Salisu et al:: Identification, Characterization and Quantification of Chemical Compounds

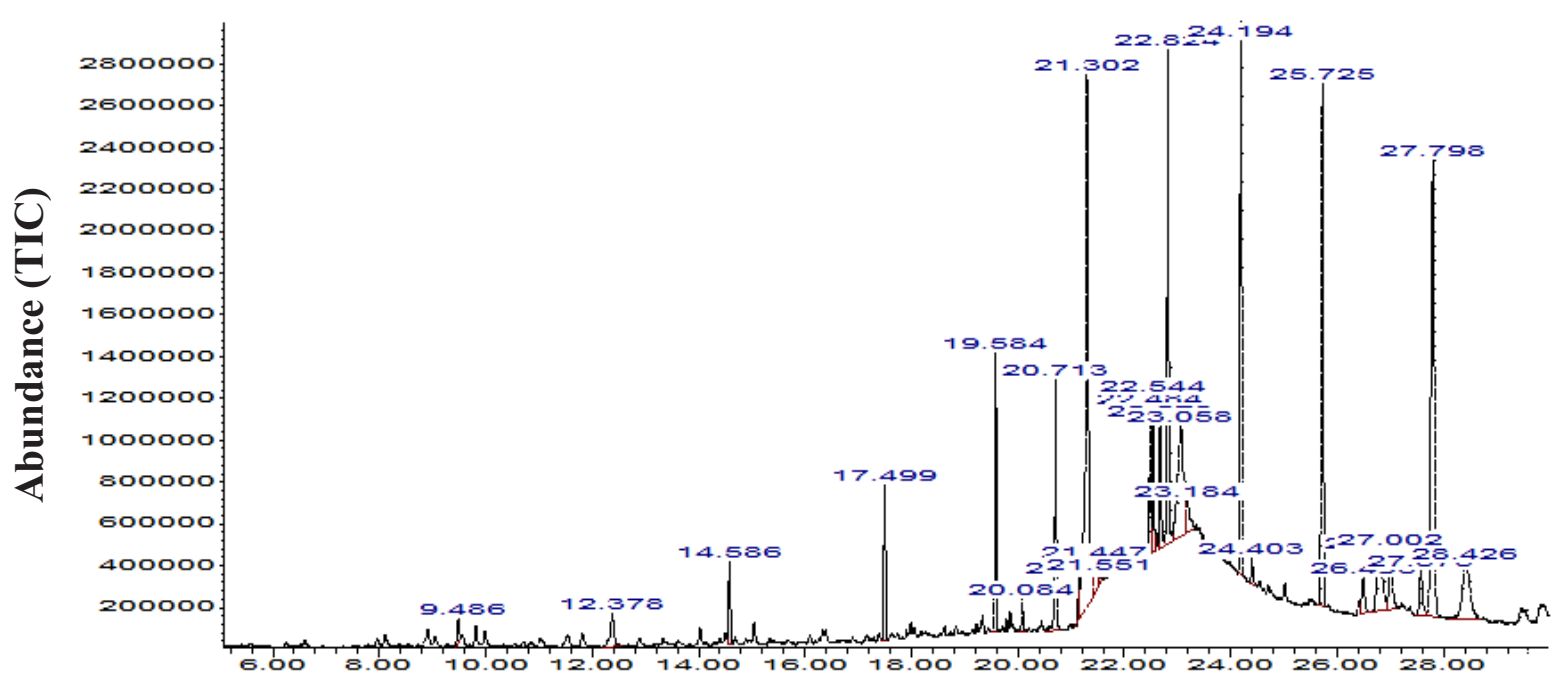

Figure 1: GC-MS Chromatogram of B. alba

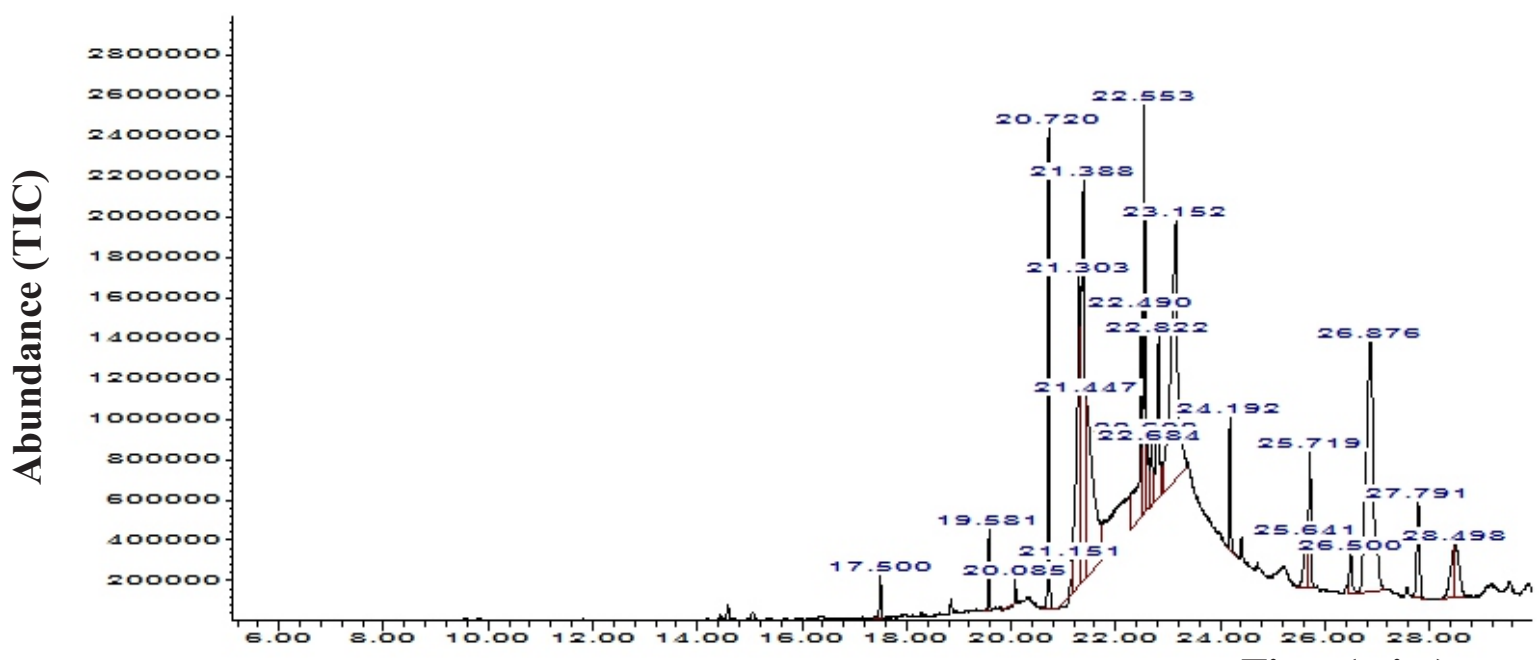

Time (min.)

Figure 2: GC-MS Chromatogram of C. crepidioides

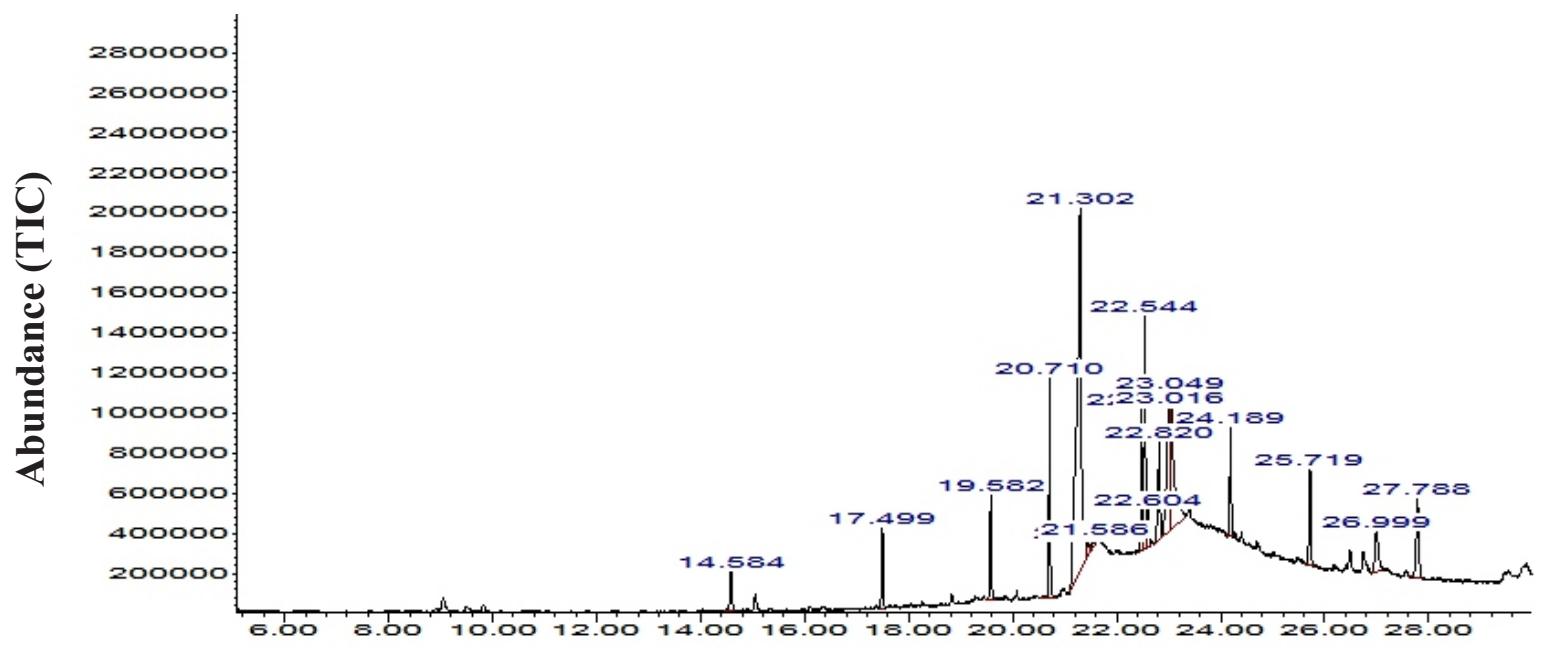

Time (min.)

Figure 3: GC-MS Chromatogram of L. taraxacifolia 
Salisu et al: Identification, Characterization and Quantification of Chemical Compounds

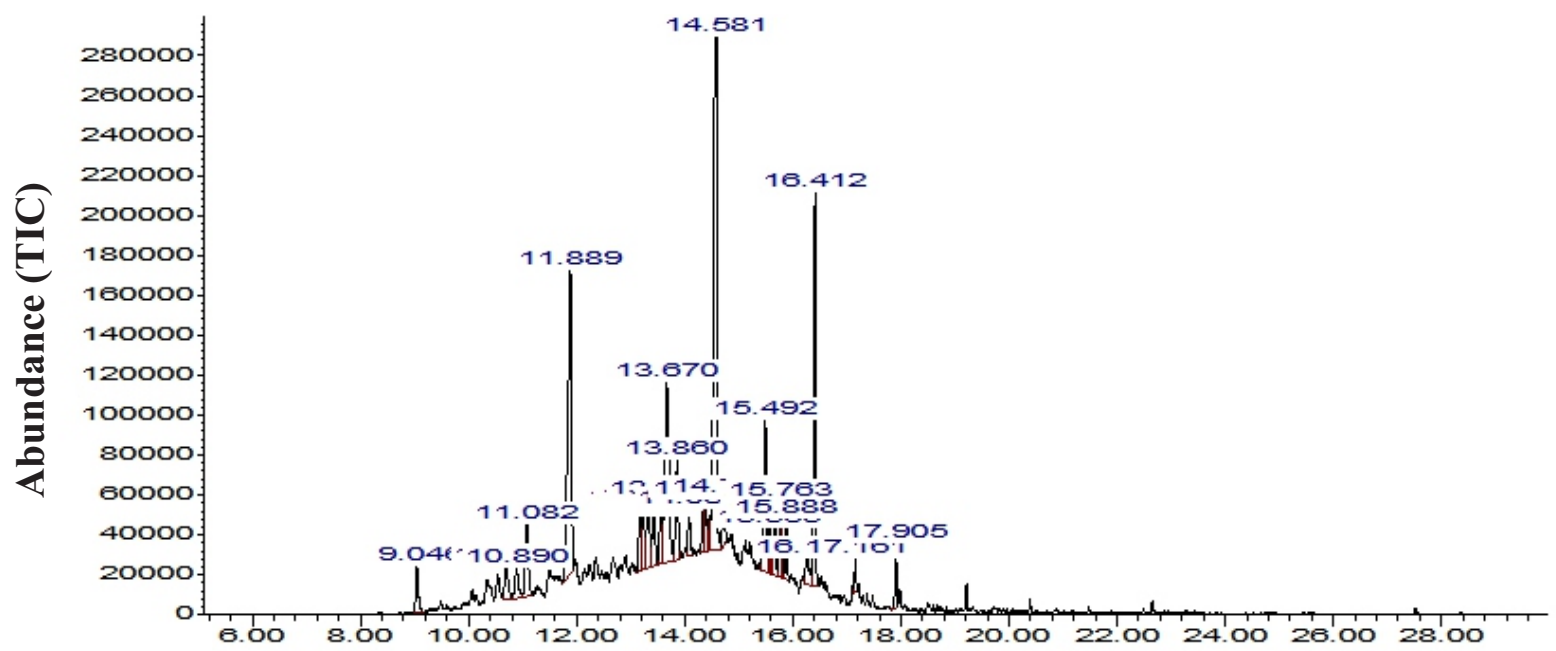

Time (min.)

Figure 4: GC-MS Chromatogram of S. biafrae

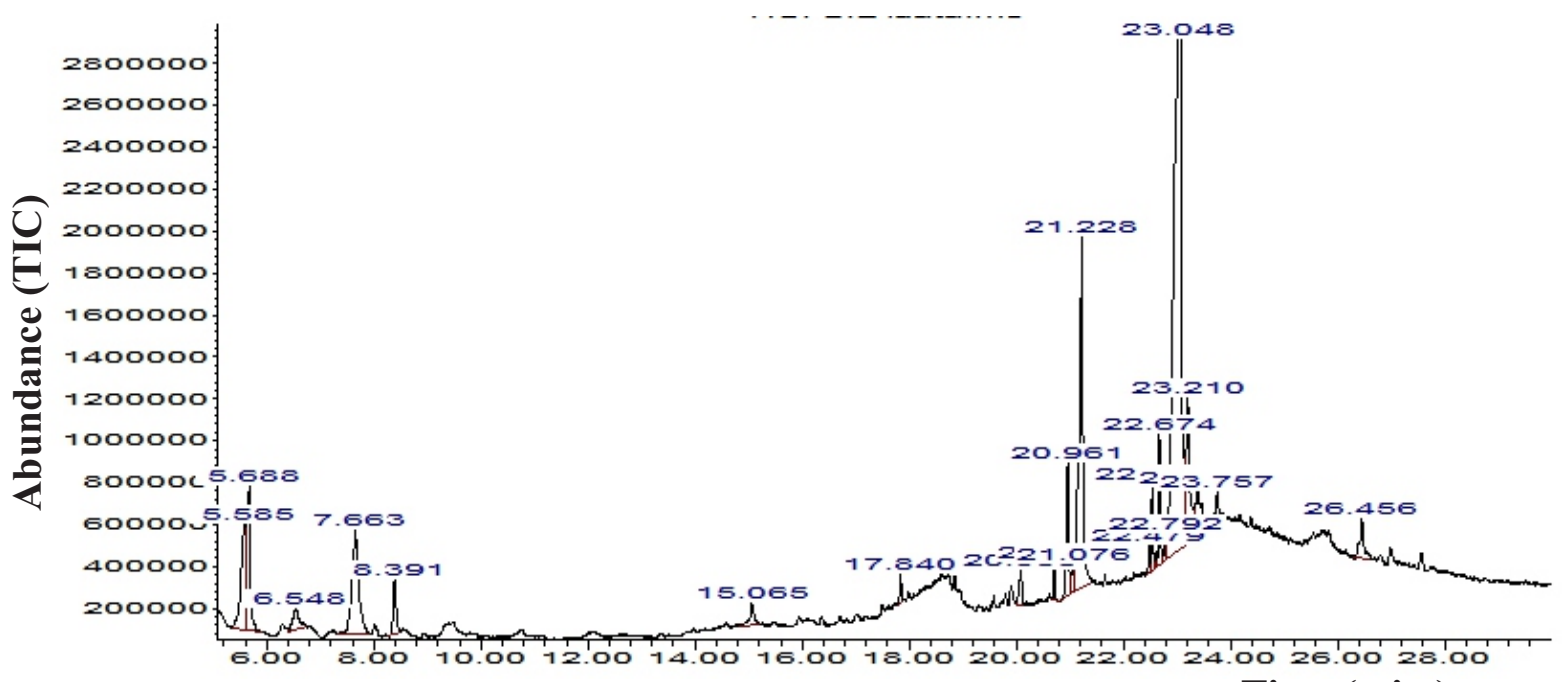

Figure 5: GC-MS Chromatogram of S. nigrum

Time (min.)

B. albaEleven phyto-components were identified from $B$. alba leaves. High quality match linoleic $(97 \%)$ and palmitic acids (98\%) were identified in B. alba composition (6.85\% and 5.47\%) (Table 1$)$. Although, $2.37 \%$ of oleic acid, $2 \%$ of phytol and $2.5 \%$ of phthalic acid were identified in B. alba but also with high quality match mass spectrum of $99 \%, 98 \%$ and $90 \%$ respectively. Small quantity of carophyllene (1.26\%) and 2, 4-decadienal (trans, trans) $(0.49 \%)$ were also identified in $B$. alba with $99 \%$ and $87 \%$ quality match respectively. Other noticeable compounds found in $B$. alba leaves are 8, 11 -octadecadienoic acid, methyl ester $(2.07 \%)$, 11,13-dimethyl-12- tetradecen-1-ol acetate $(1.04 \%), 2,3$-dihydroxypropyl elaidate (Monoelaidin) $(0.48 \%)$ and cyclotetracosane $(4.23 \%)$ (Table 1$)$. 
Table 1: Phyto-components of the Leaves of B. alba

\begin{tabular}{|c|c|c|c|c|c|c|}
\hline S/N & Name of Compound & $\begin{array}{l}\text { Molecular } \\
\text { formular }\end{array}$ & $\begin{array}{l}\text { Molecular } \\
\text { weights } \\
\text { (g·mol-1) }\end{array}$ & $\begin{array}{l}\text { Retention } \\
\text { time } \\
\text { (RT) }\end{array}$ & $\begin{array}{c}\text { Composition } \\
(\%)\end{array}$ & $\begin{array}{c}\text { Quality } \\
(\%)\end{array}$ \\
\hline 1 & 2,4-Decadienal (trans,trans) & $\mathrm{C}_{10} \mathrm{H}_{16} \mathrm{O}$ & 152.23 & 9.49 & 0.49 & 87 \\
\hline 2 & Caryophyllene & $\mathrm{C}_{15} \mathrm{H}_{24}$ & 204.36 & 12.38 & 1.26 & 99 \\
\hline 3 & $\begin{array}{l}\text { Hexadecanoic acid, methyl } \\
\text { ester (palmitic acid) }\end{array}$ & $\mathrm{C}_{17} \mathrm{H}_{34} \mathrm{O}_{2}$ & 270.45 & 20.71 & 5.47 & 98 \\
\hline 4 & $\begin{array}{l}\text { Dibutyl phthalate (phthalic } \\
\text { acid) }\end{array}$ & $\mathrm{C}_{16} \mathrm{H}_{22} \mathrm{O}_{4}$ & 278.34 & 21.14 & 2.5 & 90 \\
\hline 5 & $\begin{array}{l}\text { 8,11-Octadecadienoic acid, } \\
\text { methyl ester }\end{array}$ & $\mathrm{C}_{19} \mathrm{H}_{34} \mathrm{O}_{2}$ & 294.47 & 22.48 & 2.07 & 99 \\
\hline 6 & $\begin{array}{l}\text { 11-Octadecenoic acid, methyl } \\
\text { ester (oleic acid) }\end{array}$ & $\mathrm{C}_{19} \mathrm{H}_{36} \mathrm{O}_{2}$ & 296.49 & 22.55 & 2.37 & 99 \\
\hline 7 & Phytol & $\mathrm{C}_{20} \mathrm{H}_{40} \mathrm{O}$ & 296.53 & 22.68 & 2 & 98 \\
\hline 8 & $\begin{array}{l}\text { 9,12-Octadecadienoic acid } \\
\text { (Z,Z)- (linoleic acid) }\end{array}$ & $\mathrm{C}_{18} \mathrm{H}_{32} \mathrm{O}_{2}$ & 280.45 & 23.06 & 6.85 & 97 \\
\hline 9 & $\begin{array}{l}\text { 11,13-Dimethyl-12- } \\
\text { tetradecen-1-ol acetate }\end{array}$ & $\mathrm{C}_{18} \mathrm{H}_{34} \mathrm{O}_{2}$ & 282.46 & 23.19 & 1.04 & 91 \\
\hline 10 & $\begin{array}{l}\text { 2,3-Dihydroxypropyl elaidate } \\
\text { (monoelaidin) }\end{array}$ & $\mathrm{C}_{12} \mathrm{H}_{40} \mathrm{O}_{4}$ & 356.54 & 24.40 & 0.48 & 81 \\
\hline 11 & Cyclotetracosane & $\mathrm{C}_{24} \mathrm{H}_{48}$ & 336.64 & 28.43 & 4.23 & 98 \\
\hline
\end{tabular}

\section{C. crepidioides}

and $6.86 \%$ ) respectively. However, out of the eight Eight compounds were identified in C. identified compounds, Phthalic acid $(0.2 \%)$ was crepidioides leaves. High quality match (99\%) linoleic acid, palmitic acid and oleic acid were the major constituents identified in C. crepidioides leaves in varying composition $(14.88 \%, 27.22 \%$, the least compound identified. The remaining four compounds identified include rumenic acid $(5.16 \%)$, squalene $(1.43 \%)$, 3-eicosene $(1.81 \%)$ and hexadecane $(1.93 \%)$ (Table 2$)$.

Table 2: Phyto-components of the Leaves of C.crepidioides

\begin{tabular}{|c|c|c|c|c|c|c|}
\hline S/N & Name of Compound & $\begin{array}{l}\text { Molecular } \\
\text { formular }\end{array}$ & $\begin{array}{c}\text { Molecular } \\
\text { weights } \\
(\mathrm{g} \cdot \mathrm{mol}-1)\end{array}$ & $\begin{array}{l}\text { Retention } \\
\text { time (RT) }\end{array}$ & $\begin{array}{c}\text { Composition } \\
(\%)\end{array}$ & $\begin{array}{l}\text { Quality } \\
(\%)\end{array}$ \\
\hline 1 & $\begin{array}{l}\text { 1,2-Benzenedicarboxylic acid, bis 2- } \\
\text { methylpropyl) ester (Phthalic acid) }\end{array}$ & $\mathrm{C}_{16} \mathrm{H}_{22} \mathrm{O}_{4}$ & 278.34 & 20.08 & 0.2 & 87 \\
\hline 2 & $\begin{array}{l}\text { Hexadecanoic acid, methyl ester } \\
\text { (Palmitic acid) }\end{array}$ & $\mathrm{C}_{17} \mathrm{H}_{34} \mathrm{O}_{2}$ & 270.45 & 20.72 & 27.22 & 99 \\
\hline 3 & $\begin{array}{l}\text { Methyl 9-cis,11-trans- } \\
\text { octadecadienoate (Rumenic acid) }\end{array}$ & $\mathrm{C}_{18} \mathrm{H}_{32} \mathrm{O}_{2}$ & 280.45 & 22.49 & 5.16 & 99 \\
\hline 4 & $\begin{array}{l}\text { 11-Octadecenoic acid, methyl ester } \\
\text { (Oleic acid) }\end{array}$ & $\mathrm{C}_{19} \mathrm{H}_{36} \mathrm{O}_{2}$ & 296.49 & 22.55 & 6.86 & 99 \\
\hline 5 & $\begin{array}{l}\text { 9,12-Octadecadienoic acid (Z,Z)- } \\
\text { (Linoleic acid) }\end{array}$ & $\mathrm{C}_{18} \mathrm{H}_{32} \mathrm{O}_{2}$ & 280.45 & 23.15 & 14.88 & 99 \\
\hline 6 & $\begin{array}{l}\text { 2,6,10,14,18,22-Tetracosahexaene } \\
\text { (squalene) }\end{array}$ & $\mathrm{C}_{30} \mathrm{H}_{50}$ & 410.72 & 25.64 & 1.43 & 99 \\
\hline 7 & 3-Eicosene, (E)- & $\mathrm{C}_{20} \mathrm{H}_{40}$ & 280.53 & 28.48 & 1.81 & 94 \\
\hline 8 & Hexadecane, 1-iodo- & $\mathrm{C}_{16} \mathrm{H}_{33} \mathrm{I}$ & 352.34 & 28.50 & 1.93 & 96 \\
\hline
\end{tabular}




\section{L. taraxacifolia}

L. taraxacifolia revealed the least (4) number of compounds out of the five vegetables analysed by GC-MS (Table 3). These compounds are palmitic acid $(42.35 \%)$, linoleic acid $(20.78 \%)$, oleic acid $(7.14 \%)$ and rumenic acid (4.06\%) with 98\%, $99 \%, 99 \%$ and $99 \%$ quality match respectively (Table 3).

Table 3: Phyto-components of the Leaves of L.taraxacifolia

\begin{tabular}{|c|c|c|c|c|c|c|}
\hline $\mathrm{S} / \mathrm{N}$ & Name of Compound & $\begin{array}{r}\text { Molecular } \\
\text { formular }\end{array}$ & $\begin{array}{l}\text { Molecular } \\
\text { weights } \\
(\mathbf{g} \cdot \mathbf{m o l}-\mathbf{1})\end{array}$ & $\begin{array}{l}\text { Retention } \\
\text { time (R T) }\end{array}$ & $\begin{array}{c}\text { Composition } \\
(\%)\end{array}$ & $\begin{array}{c}\text { Quality } \\
(\%)\end{array}$ \\
\hline 1 & $\begin{array}{l}\text { Hexadecanoic acid, methyl ester } \\
\text { (Palmitic acid) }\end{array}$ & $\mathrm{C}_{17} \mathrm{H}_{34} \mathrm{O}_{2}$ & 270.45 & 20.71 & 42.35 & 98 \\
\hline 2 & $\begin{array}{l}\text { Methyl 9-cis,11-trans- } \\
\text { octadecadienoate (Rumenic } \\
\text { acid) }\end{array}$ & $\mathrm{C}_{18} \mathrm{H}_{32} \mathrm{O}_{2}$ & 280.45 & 22.48 & 4.06 & 99 \\
\hline 3 & $\begin{array}{l}\text { 11-Octadecenoic acid, methyl } \\
\text { ester (Oleic acid) }\end{array}$ & $\mathrm{C}_{19} \mathrm{H}_{36} \mathrm{O}_{2}$ & 296.49 & 22.54 & 7.14 & 99 \\
\hline 4 & 9,17-Octadecadienal, (Z)- & $\mathrm{C}_{18} \mathrm{H}_{32} \mathrm{O}$ & 264.45 & 22.60 & 1.37 & 95 \\
\hline 5 & $\begin{array}{l}\text { 9,12-Octadecadienoic acid } \\
\text { (Z,Z)- (Linoleic acid) }\end{array}$ & $\mathrm{C}_{18} \mathrm{H}_{32} \mathrm{O}_{2}$ & 280.45 & 23.01 & 19.41 & 99 \\
\hline
\end{tabular}

\section{S. biafrae}

A total of 9 phyto-components ( $72 \%$ to $92 \%$ quality match) were identified from S. biafrae leaves (Table 4). 1-undecene (25.9\%) was recorded as the most abundant compound while 1-octanol, 2-butyl-oxalic acid (1.24\%) was the least compound identified (Table 4). Other compounds identified in S. biafrae leaves include oxalic acid (11.04\%), dodecane, 2, 6, 10-trimethylheptadecane $(2.77 \%)$, hexacosane $(13.84 \%)$, tetradecane $(11.57 \%)$, 1-iodo-2-methylundecane $(1.75 \%)$, pentadecane $(9.08 \%)$ and tetracosane $(5.15 \%)$.

Table 4: Phyto-components of the Leaves of S.biafrae

\begin{tabular}{lllcccc}
\hline $\mathrm{S} /$ & Name of Compound & $\begin{array}{l}\text { Molecular } \\
\text { formular }\end{array}$ & $\begin{array}{l}\text { Molecular } \\
\text { weights } \\
\mathbf{g} \cdot \mathbf{m o l}-\mathbf{1})\end{array}$ & $\begin{array}{c}\text { Retention } \\
\text { time }(\mathrm{R} \mathrm{T})\end{array}$ & $\begin{array}{c}\text { Composition } \\
(\%)\end{array}$ & $\begin{array}{c}\text { Quality } \\
(\%)\end{array}$ \\
\hline 1 & Oxalic acid & $\mathrm{C}_{2} \mathrm{H}_{2} \mathrm{O}_{4}$ & 90.04 & 10.70 & 11.04 & 72 \\
2 & 1-Octanol, 2-butyl-oxalic acid & $\mathrm{C}_{8} \mathrm{H}_{18} \mathrm{O}$ & 130.23 & 10.89 & 1.24 & 78 \\
3 & Dodecane, 2,6,10-trimethyl- & $\mathrm{C}_{15} \mathrm{H}_{32}$ & 212.42 & 11.08 & 2.77 & 78 \\
& Heptadecane & $\mathrm{C}_{26} \mathrm{H}_{54}$ & 366.71 & 11.89 & 13.84 & 78 \\
4 & Hexacosane & $\mathrm{C}_{40} \mathrm{H}_{82}$ & 563.08 & 13.67 & 11.57 & 78 \\
6 & Tetradecane & $\mathrm{C}_{11} \mathrm{H}_{22}$ & 154.29 & 14.58 & 25.90 & 72 \\
7 & 1-Undecene & $\mathrm{C}_{12} \mathrm{H}_{25} \mathrm{I}$ & 296.23 & 15.76 & 1.75 & 72 \\
8 & Pentadecane, 2-methyl- & $\mathrm{C}_{16} \mathrm{H}_{34}$ & 226.44 & 16.41 & 9.08 & 91 \\
9 & Tetracosane & $\mathrm{C}_{24} \mathrm{H}_{50}$ & 338.65 & 17.90 & 5.15 & 78 \\
\hline
\end{tabular}

\section{S. nigrum}

S. nigrum presents the only and highest composition of linolenic acid (47.47\%) with $98 \%$ quality match out of 12 compounds identified (Table 5). However, linoleic acid was absent while other compounds identified include pure phytol $(2.23 \%)$, palmitic acid $(14.22 \%)$, stearic acid (5.93\%), 4H-pyran-4-one, 2,3- dihydro-3,5-dihydroxy-6-methyl (10.65\%), 2,6octadienal, 3,7-dimethyl-, (E) (1.7\%), benzoic acid, 4ethoxy-, ethyl ester $(1.01 \%)$; cyclododecane $(0.43 \%)$, 13-tetradece-11-yn-1-ol (0.4\%), cis-13-octadecenoic acid, methyl ester (1.39\%), E,E,Z-1,3,12nonadecatriene-5,14-diol (2.91\%) and $1 \mathrm{H}-1,2,3-$ triazolo[4,5-c]quinoline-1-hexanoic acid (1.62\%) (Table 5). 
Table 5: Phyto-components of the leaves of S.nigrum

\begin{tabular}{|c|c|c|c|c|c|c|}
\hline $\mathrm{S} / \mathrm{N}$ & Name of Compound & $\begin{array}{l}\text { Molecular } \\
\text { formular }\end{array}$ & $\begin{array}{l}\text { Molecular } \\
\text { weights } \\
(\mathrm{g} \cdot \mathrm{mol}-1)\end{array}$ & $\begin{array}{l}\text { Retention } \\
\text { time (R T) }\end{array}$ & $\begin{array}{c}\text { Composition } \\
(\%)\end{array}$ & $\begin{array}{c}\text { Quality } \\
(\%)\end{array}$ \\
\hline 1 & $\begin{array}{l}\text { 4H-Pyran-4-one, 2,3-dihydro-3,5-dihydroxy- } \\
\text { 6-methyl- }\end{array}$ & $\mathrm{C}_{6} \mathrm{H}_{8} \mathrm{O}_{4}$ & 144.13 & 5.69 & 10.65 & 86 \\
\hline 2 & 2,6-Octadienal, 3,7-dimethyl-, (E) & $\mathrm{C}_{10} \mathrm{H}_{16} \mathrm{O}$ & 152.23 & 8.39 & 1.70 & 96 \\
\hline 3 & Benzoic acid, 4-ethoxy-, ethyl ester & $\mathrm{C}_{11} \mathrm{H}_{14} \mathrm{O}_{3}$ & 194.23 & 15.07 & 1.01 & 95 \\
\hline 4 & Cyclododecane & $\mathrm{C}_{12} \mathrm{H}_{24}$ & 168.32 & 17.84 & 0.43 & 89 \\
\hline 5 & Hexadecanoic acid, methyl ester & $\mathrm{C}_{17} \mathrm{H}_{34} \mathrm{O}_{2}$ & 270.45 & 20.71 & 0.57 & 96 \\
\hline 6 & n-Hexadecanoic acid (Palmitic acid) & $\mathrm{C}_{16} \mathrm{H}_{32} \mathrm{O}_{2}$ & 256.42 & 21.23 & 13.65 & 99 \\
\hline 7 & 13-Tetradece-11-yn-1-ol & $\mathrm{C}_{14} \mathrm{H}_{24} \mathrm{O}$ & 208.34 & 22.48 & 0.40 & 96 \\
\hline 8 & cis-13-Octadecenoic acid, methyl ester & $\mathrm{C}_{19} \mathrm{H}_{36} \mathrm{O}_{2}$ & 296.49 & 22.54 & 1.39 & 97 \\
\hline 9 & Phytol & $\mathrm{C}_{20} \mathrm{H}_{40} \mathrm{O}$ & 296.53 & 22.68 & 2.23 & 99 \\
\hline 10 & $\begin{array}{l}\text { 9,12,15-Octadecatrienoic acid, (ZZ,Z)- } \\
\text { (Linolenic acid) }\end{array}$ & $\mathrm{C}_{19} \mathrm{H}_{32} \mathrm{O}_{2}$ & 292.46 & 23.05 & 47.47 & 98 \\
\hline 11 & Octadecanoic acid & $\mathrm{C}_{18} \mathrm{H}_{36} \mathrm{O}_{2}$ & 284.48 & 23.208 & 5.93 & 94 \\
\hline 12 & E,E,Z-1,3,12-Nonadecatriene-5,14-diol & $\mathrm{C}_{19} \mathrm{H}_{34} \mathrm{O}_{2}$ & 294.47 & 23.386 & 2.91 & 91 \\
\hline 13 & $\begin{array}{l}\text { 1H-1,2,3-Triazolo[4,5-c]quinoline-1- } \\
\text { hexanoic acid }\end{array}$ & $\mathrm{C}_{15} \mathrm{H}_{16} \mathrm{~N}_{4} \mathrm{O}_{2}$ & $28 / 4.32$ & 26.458 & 1.62 & 95 \\
\hline
\end{tabular}

Table 6: Some Important Bioactive Components Identified in the Five Selected Vegetables Extracts by GCMS and their Biological Activities

\begin{tabular}{|c|c|c|c|c|c|c|c|}
\hline $\begin{array}{l}\mathrm{S} / \\
\mathrm{N}\end{array}$ & Name of Compound & $\mathrm{BA}$ & $\begin{array}{l}\mathrm{C} \\
\mathrm{C}\end{array}$ & LT & SB & $\mathrm{SN}$ & $\begin{array}{l}\text { Nature of compounds/**Biological } \\
\text { Activities }\end{array}$ \\
\hline 1 & Linoleic Acid & + & + & + & - & + & $\begin{array}{l}\text { Polyunsaturated omega- } 6 \text { fatty acid. /anti- } \\
\text { inflammatory and antioxidant }\end{array}$ \\
\hline 2 & Palmitic acid & + & + & + & - & + & $\begin{array}{l}\text { Saturated fatty acids / Antioxidant, anti- } \\
\text { atherosclerotic and hypocholesterolemic }\end{array}$ \\
\hline 3 & Rumenic acid & - & + & + & - & - & Conjugated linoleic acids / Antioxidant \\
\hline 4 & Oleic acid & + & + & + & - & - & $\begin{array}{l}\text { Fatty acid / Anti-inflammatory and } \\
\text { hypocholesterolemic }\end{array}$ \\
\hline 5 & Phthalic acid & + & + & - & - & - & Aromatic dicarboxylic acid/Antioxidant \\
\hline 6 & Phytol & + & - & - & - & + & Acyclic diterpene alcohol / Anti-inflammatory \\
\hline 8 & $\begin{array}{l}\text { 4H-Pyran-4-one, 2,3- } \\
\text { dihydro-3,5-dihydroxy- } \\
\text { 6-methyl }\end{array}$ & - & - & - & - & + & Flavonoid fraction / Anti-inflammatory \\
\hline 9 & Oxalic acid & - & - & - & + & - & Organic dicarboxylic acid / Antioxidant \\
\hline
\end{tabular}

$\mathrm{BA}=$ Basella alba, $\mathrm{CC}=$ Crassocephalum crepidioides, $\mathrm{LT}=$ Launaea taraxacifolia, $\mathrm{SB}=$ Senecio biafrae, $\mathrm{SN}=$ Solanum nigrum,

+ present, - absent

**Source: Dr. Duke's phytochemical and ethnobotanical database (Duke, 2014)

Important Antioxidant Compounds and their Biological Activities in the Vegetables Some important antioxidant compounds such as phytol, linolenic, linoleic, palmitic, oleic, stearic, oxalic and phthalic acids were identified in the leaves of all plants with a substantial percentage abundance (quantity) and quality match ranging from $72 \%$ to $99 \%$. Summary of important identified compounds with their biological activities in each vegetable extract are presented in table 6 . 


\section{DISCUSSION}

Analysis of Bioactive Components of Vegetables' Extracts by GC-MS

GC-MS analysis separates all the volatile components in the wild vegetable samples and presents a characteristic spectral output. A total of 44 compounds were subsequently identified in this study. Some major important fatty acids (linolenic acid, linoleic acid, palmitic acid, rumenic acid, stearic acid, oleic acid, phytol and oxalic acid) that were identified in the plants are beneficial to heart health (Okpuzor and Salisu, 2015; Bourourou et al., 2016).Most of these phytoconstituents have been reported to display remarkable biological activity against certain diseases, prevention of many diseases and health promoting properties (Olowokudejo et al., 2008; Aboul-Enein et al., 2014). These essential fatty acids cannot be synthesized in the body unlike most fats, but can be found in many vegetables with abundance in wild plants (Bhardwaj et al., 2016). The presence of linoleic and linolenic acids in 4 out of the 5 selected wild edible vegetables in this study support earlier reports of Melariri et al., (2012) and Bhardwaj et al., (2016).

However, percentage composition of linolenic acid in S. nigrum leaves is $47.1 \%$. This area percentage is higher than that reported by Padmashree et al., (2014) in India, where the area percentage of linolenic acid in $S$. nigrum leaves was $0.41 \%$. Also, our result revealed absence of linoleic acid $(0 \%)$ in contrast to $59.1 \%$ of linoleic acid obtained by Padmashree et al., (2014) in $S$. nigrum leaves. This may be attributed to disparity in composition of the soil, temperature, water content and other environmental factors that may affect the bioavailability of different compounds in the leaves. Although 13.9\% palmitic acid and $3.6 \%$ stearic acid from the same leaves (S. nigrum leaves) obtained in this study were almost consistent with $14.22 \%$ palmitic acid and 5.93\% stearic acid reported by Padmashree et al., (2014).

Linoleic acid belongs to omega 6-fatty acids used in the biosynthesis of arachidonic acid and thus some prostaglandins, thromboxane and leukotrienes collectively known as eicosanoids. Linoleic acid (octadecadienoic acids) is a polyunsaturated fatty acid that plays a key role in support of heart vitality by lowering LDL cholesterol and reduces risk of developing heart disease (Farvid et al., 2014). Linolenic acid (octadecatrienoic acid) is an essential fatty acid convertible in-vivo to omega-3 fatty acids (Seeley et al., 2011). These two different essential fatty acids (linoleic and linolenic acids) are not produced in humans but usually found in plants (Melariri et al., 2012). Due to the health benefits associated with them, they are marketed as health supplements (Gaullier et al., 2005). They have been reported to be beneficial in the prevention and management of coronary heart disease and other chronic diseases (Bourourou et al., 2016).

It has been suggested that a combination of palmitic and linoleic acids displays antioxidant properties and can help prevent atherosclerosis (underlying pathogenesis of myocardial infarction) in rats (Cho et al., 2010). A combination of palmitic and linoleic acids was identified in 3 out of the 5 selected vegetables except in S. biafrae and S. nigrum leaves (Table 6). Increased antioxidant activities in myocardial infarction- induced experimental rats pretreated with these wild vegetables further confirm their antioxidant properties (Okpuzor and Salisu, 2015). Conjugated form of linoleic acid known as rumenic acid is majorly found in meat and dairy products (Gnadig et al., 2003). This fatty acid (rumenic acid) was also found in L. taraxacifolia and C. crepidiodes leaves. This is in agreement with the earlier study of Aberoumand (2009) which showed that conjugated linoleic acids are available in edible wild plants. Rumenic acid has been documented as antioxidative (Flintoff-Dye and Omaye, 2005), anticarcinogenic (Agnieszka et al., 2010), antiatherogenic (Valeille et al., 2005) and immune response modulators (Eder et al., 2005).

A saturated fatty acid (SFA), stearic acid, was present only in $S$. nigrum. Its hypocholesterolemic properties had been associated with low density lipoprotein (LDL) cholesterol levels (Mensink, 2005). Baskaran et al., (2015) reported oleic acid (omega-9) as antiinflammatory compound and high quality oleic 
acid (99\% quality match) was identified in B. alba, C. crepiodiodes and L. taraxacifolia. This is in agreement with Kazadi et al. (2014) who pointed that wild plants are potential new sources of oleic acids.

Although, oxalates was reported as antinutrient in vegetables (Citation et al., 2013)., as natural antioxidant identified in only $S$. biafrae in this study, has been reported to suppress lipid peroxidation (Kayashima and Katayama, 2002). Phytol is another valuable compound documented in ethnobotanical database as natural antioxidant. This compound was present in $B$. alba $(2 \%)$ and S. nigrum $(2.3 \%)$ but absent in other vegetables. This result was not in agreement with $6.3 \%$ of phytol reported by Owokotomo et al. (2012) in C. crepidioides leaves. This disparity may be attributed to different geographical and climatic conditions associated with varying composition in the leaves. All these identified natural compounds possess antioxidant, atherosclerotic, anti-inflammatory and hypocholesterolemic properties that support previous studies and thus could serve as potent compounds for mitigation of myocardial infarction and can also found uses in drug development (Sheela and Uthayakumari et al., 2013; Salisu et al., 2014).

In conclusion, these indigenous vegetables with high antioxidants can be incorporated into our daily diet as functional foods for nutritional improvement and most importantly to prevent diseases. Furthermore, the conservation / preservation of the biodiversity of underutilized indigenous vegetables should be undertaken. Isolation of the specific active compound present in the plant extract is required for further medicinal research at the molecular level.

\section{CONFLICT OF INTEREST}

The authors have not declared any conflict of interest

\section{ACKNOWLEDGEMENTS}

Central Research Laboratory, University of Lagos Akoka, Lagos, Nigeria is acknowledged for GCMS facility

\section{FUNDING}

This research did not receive any specific grant from funding agencies in the public, commercial, or not-for-profit sectors.

\section{REFERENCES}

Aberoumand, A. (2009). Identification of fatty acids in edible wild plants by gas chromatography. Food Analytical Methods, 2 (3): 208-211.

Aboul-Enein, A., Abu, E.F., Shalaby E. and ElShemy, H. (2014). Potent anticancer and antioxidant activities of active ingredients separated from Solanum nigrum and Cassia italica extracts. Journal of Arid Land Studies, 24(1): 145-152

Adebayo, A. G. (2009). Inventory of antidiabetic plants in selected districts of Lagos State, Nigeria. Journal of Ethnopharmacology, 121(1):135-139.

Adebooye, O. C. and Ajayi, O. A. (2008). Future of the Nigerian under-exploited indigenous fruits and vegetables in the era of climate change: The Need for farmers Education. Tropentag 2008 Conference on International Research on Food Security, Natural Resource Management and Rural Development, University of Hohenheim, Held between $7^{\text {th }}$ and $9^{\text {th }}$ October, 2008. pp 1-4.

Ahmad, A., Alkarkhi, A. F. M., Hena, S. and Kim, L. H. (2009). Extraction, separation and identification of chemical ingredients of Elephantopus Scaber L.using factorial design of experiment. International Journal of Chemistry, 1:1-14.

Agnieszka, B., Andrzej, T., Agnieszka, D., Weronika, K. and Wojciech, B. (2010). Influence of diet enriched with conjugated linoleic acids on their distribution in tissues of rats with DMBA induced tumors. Lipids in Health and Disease, 9: 126.

Amujoyegbe, B. J., Oyedele, D. J., Idowu, M. K., Ayinde, J. O. and Adebooye, O. C. (2015). On-farm adoption of under-utilized indigenous vegetable production among small holder farmers in Nigeria: Implication for economic empowerment 
and genetic conservation. Journal of Agricultural Extension and Rural Development, 9:283-289.

Arawande, J.O., Amoo, I.A. and Lajide, L. (2013) Chemical and phytochemical composition of wild lettuce Launaea taraxacifolia. Journal of Applied Phytotechnology in Environmental Sanitation, 2: 25-30.

Ayodele, A. E. (2005). The medicinally important leafy vegetables of south-western Nigeria Ethnobotanical leaflets. Accessed 2 May 2013 via http://www.siu.edu/ ebl/ leaflets/ayodele.htm

Bamidele, O., Akinnuga, A. M., Olorunfemi, J. O., Odetolo, O. A., Oparaji, C. K. and Ezelgbo, N. (2010). Effects of aqueous extract of Basella alba leaves on haematological and biochemical parameters in Albino rats. African Journal of Biotechnology, 9: 6952-6955.

Baskaran, G., Salvamani, S., Ahmad, S.A., Shaharuddin, N.A., Pattiram, P.D. and Shukor, M.Y. (2015). HMG-CoA reductase inhibitory activity and phytocomponent investigation of Basella alba leaf extract as a treatment for hypercholesterolemia. Drug Design and Development Therapy, 9:509-517.

Bhardwaj, K., Verma, N., Trivedi, R.K., Bhardwaj, S. and Shukla, N. (2016). Significance of ratio of omega-3 and omega-6 in human health with special reference to flaxseed oil. International Journal of Biological Chemistry, 10:1-6.

Bourourou, M., Heurteaux, C. and Blondeau, N. (2016). Alpha-linolenic acid given as enteral or parenteral nutritional intervention against sensorimotor and cognitive deficits in a mouse model of ischemic stroke. Neuropharmacology, 108: $60-72$.

Cho, K. H., Hong, J. H. and Lee, K.T. (2010). Monoacylglycerol (MAG)-oleic acid has stronger antioxidant, anti-atherosclerotic, and protein glycation inhibitory activities than MAG-palmitic acid. Journal of Medicinal Food, 13(1): 99-107

Citation, R. M., Alcantara, R. M., Hurtada, W.
A.and Dizon, E. I. (2013). The nutritional value and phytochemical components of Taro [Colocasia esculenta (L.) Schott] Powder and its Selected Processed Foods. Journal of Nutrition E Food Sciences, 3: 207.

Dansi, A., Adjatin, A., Adoukonou-Sagbadja, H., Faladé, V., Yedomonhan, H., Odou, D. and Dossou, B. (2008) Traditional leafy vegetables and their use in the Benin Republic. Genetic Resources and Crop Evolution, 55, 1239-1256.

Duke, J.A. (2014). Phytochemical and ethnobotanical databases. Accessed 27 Nov., 2014 from<http://search. conduit.com $/$ results.aspx?q=dr+duke's + phytochemical + ethnobot anical+databases\&ctid>

Eder, K., Slomma, N., Becker, K. and Brandsch, C. (2005) Effects of linseed oil supplementation on concentration of (n-3) polyunsaturated fatty acids in liver phospholipids of rats fed diets containing either an oil rich in conjugated linoleic acids, sunflower oil or high-oleic sunflower oil. Journal of Animal Physiology and Animal Nutrition, 89:45-54.

Farvid, M. S., Ding, M., Pan, A., Sun, Q., Chiuve, S. E., Steffen, L. M., Willett, W. C. and Hu, F. B. (2014). Dietary linoleic acid and risk of coronary heart disease: A systematic review and meta-analysis of prospective cohort studies. Circulation, 130(18):1568-1578.

Flintoff-Dye, N. L. and Omaye, S.T. (2005) Antioxidant effects of conjugated linoleic acid isomers human low-density lipoproteins. Nutrition research, 25:1-12

Gaullier, J. M., Halse, J., Hoye, K., Kristiansen, K., Fagertun, H., Vik, H. and Gudmundsen, O. (2005). Supplementation with conjugated linoleic acid for 24 months is well tolerated by and reduces body fat mass in healthy overweight humans. The Journal of Nutrition, 135: 778-784.

Gnadig, S., Xue, Y., Berdeaux, O., Cheardigni, J. M. and Sebe-dio, J. L. (2003). Conjugated linoleic acid (CLA) as a functional ingredient. In: Saadholm TM, Saar-ela M 
(eds) Functional dairy products. CRC, Cambrige, pp 263-297

Janick, J. (2011). Horticultural review. John and Willey and Co. 38: 305.

Kazadi, M., Bokota, M.T. and Mpiana, P.T. (2014). Potential new sources of oleic acids from wild plants from Kivu, D.R. Congo. Journal of Physical and Chemical Sciences, 1(2): 1-4.

Kayashima, T. and Katayama, T. (2002). Oxalic acid is available as a natural antioxidant in some systems Biochimica et Biophysica Acta, 1573(1):1-3.

Kumar, V., Bhat, Z. A., Kumar, D., Bohra, P. and Sheela, S. (2011). In vitro antiinflammatory activity of leaf extracts of Basella alba Linn. var. alba. International Journal of Drug Development and Research, 3:176-179.

Lienou, L. L., Telefo, P.B., Bayala, B., Yemele, M.D., Lemfack, M.C., Mouokeu, C., Goka, C.S., Tagne, S.R. and Moundipa, F.P. (2010). Effect of ethanolic extract of Senecio biafrae on puberty onset and fertility in immature female rat. Cameroon Journal of Experimental Biology, 6:101-109.

Melariri, P., Campbell, W., Etusim, P. and Smith, P. (2012). In Vitro and in Vivo antimalarial activity of linolenic and linoleic acids and their methyl esters. Advanced Studies in Biology, 4(7):333-349.

Mensink, R.P. (2005). Effects of stearic acid on plasma lipid and lipoproteins in humans. Lipids, 40: 1201-1205.

Nnamani, C. V., Oselebe, H. O. and Okporie, E. O. (2010). Aspect of ethnobotany of traditional leafy vegetables utilized as human food in rural tropical communities. Animal Research International, 7(1): 1110-1115.

Ng, X.N., Chye, F.Y. and Mohd, I. A. (2012). Nutritional profile and antioxidative properties of selected tropical wild vegetables. International Food Research Journal, 19(4): 1487-1496.

Obi, R.K. (2011). Antiviral potential of vegetables: Can they be cost-effective agents for human disease? In: Gerald J., Watson R., Preedy V. (eds) Nutrients,
Dietary Supplements, and Nutriceuticals. Nutrition and Health. Humana Press Part 5, 259-276.

Odukoya, O. A., Inya-Agha, S. I., Segun, F. I., Sofidiya, M. O. and Ilori, O. O. (2007). Antioxidant activity of selected Nigerian green leafy vegetables. American Journal of Food Technology, 2:169-175.

Ogunwusi, A.A. and Ibrahim, H.D. (2016). Industrial potential of underutilized plants in Nigeria. Journal of Natural Sciences Research, 6(6):20-28

Okpuzor, J. and Salisu, T. (2015). Antioxidant enzymes activities and lipid peroxidation of aqueous extracts of selected vegetables in isoproterenol-induced myocardial infarction in male wistar albino rats. The journal of the Federation of American Societies for Experimental Biology, 29(1): 716.

Olowokudejo, J. D., Kadiri, A. B. and Travih, V.A. (2008). An ethnobotanical survey of herbal markets and medicinal plants in lagos state of Nigeria. Ethnobotanical Leaflets, 12: 851-865.

Osewa, S. O., Alamu, O., Adetiloye, I. S., Olubiyi, M. R. and Abidogun, E. A. (2013). Use of some neglected and underutilized plant species among rural dwellers in Akinyele local government area of Oyo State. Greener Journal of Agricultural Science, 3(12): 817-822.

Owokotomo, I. A., Ekundayo, O., Oladosu, I. A. and Aboaba, S. A. (2012). Analysis of the essential oils of leaves and stems of Crassocephalum crepidioides growing in south western Nigeria. International Journal of Chemistry, 4(2): 34-37

Padmashree, A., Sharma, G.K., Semwal, A.D. and Mahesh, C. (2014). Antioxygenic activity of Solanum nigrum L. leaves in sunflower oil model system and its thermal stability, Food and Nutrition Sciences, 5 (11): 8pp

Salisu, T.F., Ottu, B., Okpuzor, J. (2014). Histopathologic studies of aqueous extracts of five selected local edible vegetables in isoproterenol-induced myocardial infarction in male Wistar albino rats. Planta Medica, 80:2-39. 
Seeley, R., VanPutte, C., Regan, J. and Russo, A. (2011). Fatty Acids and blood clotting, nutrition, metabolism and temperature regulation. Seeley's Anatomy and Physiology. $9^{\text {th }}$ Edition. New York. McGraw Hill Companies Inc. 926.

Shabu, T. and Uchi, T.S. (2013). Residents' perception of environmental quality in judges quarters of Makurdi, Nigeria. Journal of Human and Social Science Research, 1 (1):39-49.

Sheela, D. and Uthayakumari, F. (2013). GC-MS analysis of bioactive constituents from coastal sand Dune taxon-Sesuvium portulacastrum (L.). Bioscience Discovery, 4(1):47-53.

Smith, F.I. and Eyzaguirre, P. (2007). African leafy vegetables: Their role in the world health organization's global fruit and vegetables initiative. African Journal of Food Agriculture Nutrition and Development, 7(3): 1-17

Stamp, P., Messmer, R. and Walter, A. (2012). Competitive underutilized crops will depend on the state funding of breeding programmes: An opinion on the example of Europe. Plant Breeding, 131: 461-464.

Suguna, J., Thenmozhi, S., Parimalam, K., Kalaiselvi, K. and Panneer selvam, K. (2015). Antimicrobial and antioxidant activity of the leaf extract of Basella alba. International Journal of Pharmacy and Pharmaceutical Research, 3 (2): 6677.

Tomoyuki, K., Yoko, A., Chika, M., Manami, M., Chiho, I., Shizuka, K. and Toshio I. (2005). Free radical scavenging and hepatoprotective actions of the medicinal herb, Crassocephalum crepidioides from the Okinawa Island. Biological and Pharmaceutical Bulletin, 28(1): 19-23

Valeille, K., Férézou, J. and Amsler, G. (2005). A cis-9, trans-11-conjugated Linoleic acidrich oil reduces the outcome of atherogenic process in hyperlipidemic hamster. American Journal of Physiology-Heart and Circulatory Physiology, 289: 652-659. 